\title{
Testing and adjusting for publication bias
}

\section{Anders Pape Møller and Michael D. J ennions}

\begin{abstract}
How can the scientific literature provide unbiased conclusions if it represents a biased sample of available studies? Publication bias refers to phenomena arising from bias in submitting, reviewing, accepting and publishing scientific results. Direct and indirect methods for investigating publication bias are now readily available, but indirect methods are generally open to altemative interpretations. Publication bias distorts attempts to review a scientific field quantitatively if the likelihood of locating completed studies depends on the strength or direction of the findings of quantitative studies. It is the responsibility of researchers, reviewers and editors to address issues of bias to ensure the existence of an unbiased literature.
\end{abstract}

PUBLICATION BIAS (PB; seeGlossary) occurs when the published literature does not reflect finished research projects in a particular subject area. If the objective of a science, such as ecology or evol utionary biology, is to makegeneralizations about the organization of theliving world and how this has arisen, then any BIAs in our knowledgecan distort our view. Moregenerally, DISSEMINATION BIAS affects our ability to retrieveinformation on completed studies and review a scientificfiel d whenever the time-lag, journal, language, data baseindexing, citation rate, public prominenceand even likelihood of publication depend on the strength or direction of scientific findings ${ }^{1-4}$.

Two aspects of PB with special relevance to biology arechoice of research topics and samplesizes. First, most biologists liveand work in temperateregions, whereas most species aretropical; most animals are beetles, but fiel d biologists are usually ornithol ogists. Published studies ther efore reflect taxonomic diversity inaccurately. Second, META-ANALYSIs involves weighting EFFECT SIZE by samplesize. In ecology and evolutionary biology, however, samplesizes might not be random with respect to the taxonomy or thebiology of the species. Largesamplesizes are usually associated with naturally common, smaller-bodied species, such as insects. Species abundance varies with latitude and habitat type, rarity and body size areal so correl ated with many aspects of life history, physiology and behaviour. Although we do not consider these potentially confounding correlations further in this review, they affect both narrativeand meta-analysis reviews and do deserve greater attention.

Therehas been a recent increase in the application of quantitative assessment of scientific publications through meta-analysis ${ }^{5-7}$. Since 1976 (Ref. 8), this has becomethe main method of assessing a body of literature in thesocial and medical sciences.
Meta-analysis summarizes a body of literature on a subject by transforming test statistics into a common metric (effect size, which is a standardized measure of the strength of a relationship between two variables of interest), and thesequantitative data aresubsequently analysed. Meta-analysis has an advantage over simpleverbal reviews because unbiased estimates of the overall strength of a hypothetical relationship can beobtained, heterogeneity in research findings identified and new research questions developed. Meta-analysis results in an increase in knowledge. Although only recently introduced to ecology ${ }^{7}$, an increase in the use of metaanalysis in this field is clear from bibliographic data bases [ five meta-analyses (1996); six (1997); eight (1998); 19 (1999)].

\section{What is publication bias?}

Meta-analysis is based on theassumption that the literature reviewed is unbiased ${ }^{5,6}$. If thetotal number of studies that have been conducted as the source of all publications is considered, only somestudies will bewritten up and submitted for publication, a fraction of those will besubmitted by editors for refereeing, a fraction of those will berecommended by referees for publication, and a fraction of those will be published. Publication bias occurs whenever the strength or direction of theresults of published and unpublished studies differ. Although experimental quality, methodological rigour, level of replication (and social factors, such as author or institute prestige) can increase the probability of publication, they do not necessarily give riseto PB. Therewill be no bias if published articles are a random sample of the original source pool of research with respect to research findings. Given the various potential biases, authors, reviewers and editors could all be responsible for creating a biased scientific literature. Whether that is the case is thetopic of this review. Unfortunately there arelittlespecific data on PB in ecological or evolutionary research, and wether efore draw our examples primarily from the medical literature.

Since 1979 (Ref. 9), the meta-analysis literature in medical and social sciences is repletewith discussions of bias from thelevel of choice of research topics, to submission, publication and citation. I ndeed, several meta-analyses haveaddressed these issues and even attempted to assess the potential effect ${ }^{10}$. By contrast, not onenarrativereview in themedical sciences 


\section{Box 1 Fail-safe numbers}

Fail-safe or file-drawer methods exploit the fact that an average effect size, adjusted for sample size, has a given associated significance level. A larger number of unpublished null results would be needed to nullify the significance of an analysis with a highly significant mean effect. Rosenthala recommended the use of Eqn I to estimate the fail-safe number of unpublished results $(X)$ :

$$
X=\left(\Sigma Z_{\mathrm{j}}\right)^{2} / 2.706-K
$$

where $Z_{\mathrm{j}}=Z_{\mathrm{rj}} \sqrt{ }\left(N_{\mathrm{j}}-3\right), Z_{\mathrm{rj}}$ is Fisher's z-transformed correlation coefficient for the relationship between the two variables of interest for sample $j, N_{\mathrm{j}}$ is sample size for sample $j$ and $K$ is the number of analysis units (depending on the level of analysis, this can be the number of samples, studies or species). The value 2.706 is based on a one-tailed $P$ value of 0.05 . The failsafe number estimates the number of studies that are unknown to us as a result of either journals rejecting papers with null results or scientists not writing up null results. A different way of viewing the fail-safe number is that it provides an estimate of the number of future studies needed to change a significant effect to a nonsignificant one. A fail-safe number of $5 K+10$ was considered to provide evidence of a robust average effect sizea.

In a study of survivorship of males in relation to expression of secondary sexual characters, J ennions et al. ${ }^{\text {b }}$ found an average Pearson product-moment correlation coefficient adjusted for sample size of 0.125 based on studies of 40 species. Entering these values in the equation gives a fail-safe number larger than 1200 , which by far exceeds $5 \times 40+10=210$.

\section{References}

a Rosenthal, R. (1991) Meta-analytic Procedures for Social Research, Sage

b J ennions, M.J .et al. (2001) Sexually selected traits and adult survival: a meta-analysis. Q. Rev. Biol. 76, 3-36

mentioned PB. Examination of the past decade of Annual Reviews of Ecology and Systematics, Biological Reviews and Quarterly Review of Biol ogy leaves thesame impression: narrativereviews rarely address whether theliterature is biased. When they do, the effect of PB is never quantified. Hence, metaanalysis has yet another advantage over simple narrativereviews by quantitatively assessing potential biases.

Recently, several studies in ecology and evolution have suggested that PB might have tainted our view of a given field ${ }^{11-16}$. Thereareat least three levels in which PB occurs: submission, review and editorial decisions.

\section{Submission bias}

SUBMISSION BIAS has been studied little because it requires information on the source population of the research undertaken. A typical method to investigate this bias is to contact prospectiveauthors and members of academic societies to obtain a sample of potential papers. For example, a survey of 1000 psychologists showed that authors were morelikely to submit studies that refuted a null hypothesis than those studies that did not (66\% versus $22 \%)^{1}$. Many other studies have found similar or even higher rates of lack of submission because investigators did not consider their results to beinteresting ${ }^{2}$ or becausethe results were 'inexplicable' (i.e. inconsistent with mainstream theory).

Although these studies suggest that submission bias related to the strength of research findings is sometimes important, wecannot besure. The data are often based on self-reporting, and the investigators surveyed are usually sel ected nonrandomly. Even so, investigators do appear to bea major source of PB.

\section{Editorial bias}

Disappointed authors often blame editors, or explicit or implicit journal policies, as a key cause of PB (Ref. 3). Editors haveto present original and interesting findings tothereadership, so old, wellknown null results rarely get prominent coverage. Authors know this: the presence of the word 'novel' in titles and abstracts has increased exponentially in recent years ${ }^{17}$ ! A questionnairestudy of 429 editors or editorial board members for 19 leading journals in management showed that thelikelihood of acceptance was strongly increased by thereputation of theauthor, successful testing of theauthor's new theory, and originality. Nonsignificant results, replication and lack of novelty reduced the probability of publication ${ }^{18}$. I n biology, replication of a study is so broadly defined that testing for the samerelationship in another species or phylum can still be classified as replication. Theseattitudes strongly discourage authors from replicating studies.

Editorial policies that requiregreater statistical power when results are not significant can also bias the literature because increasing samplesizes delays publication. It might even discourage researchers from tackling certain topics if they suspect that the null hypothesis is correct and previously published statistically significant findings are simply a result of chance. Finally, editorial bias can al so arise when referees of manuscripts are not chosen blindly with respect to author affiliation, institutional prestigeor research findings.

\section{Reviewer bias}

Reviewer bias based on author prestige, nationality or gender could influence which manuscripts get published and in which journals. Several studies investigating peer-reviewer effects on PB show that inter-reviewer agreement is generally poor and biased by the reviewers' own research findings ${ }^{19,20}$. The prestige of theinstitution of theauthors has been shown to belinked to the probability of recommendation for acceptance of brief reports, but not for full papers, in theJ ournal of Pediatrics ${ }^{21}$.

Link $^{22}$ found that US reviewers of manuscripts for a US medical journal weremorelikely torecommend acceptance of US than they did nonUS manuscripts. US reviewers ranked US papers much more favourably than nonUS papers, whereas the difference for nonUS reviewers was nonsignificant. In 


\section{Box 2. The funnel graph}

Fig. I (a)

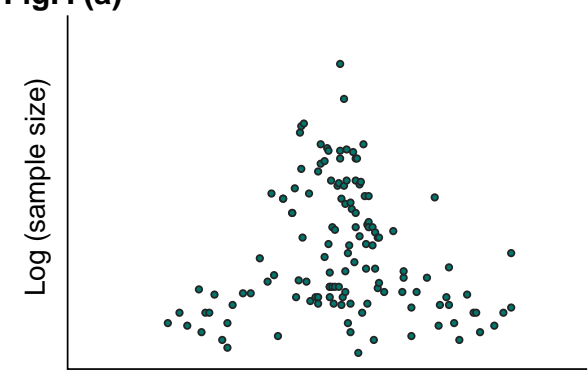

Effect size (b)

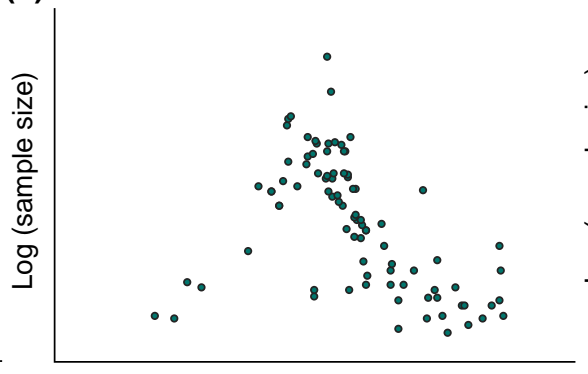

Effect size (c)

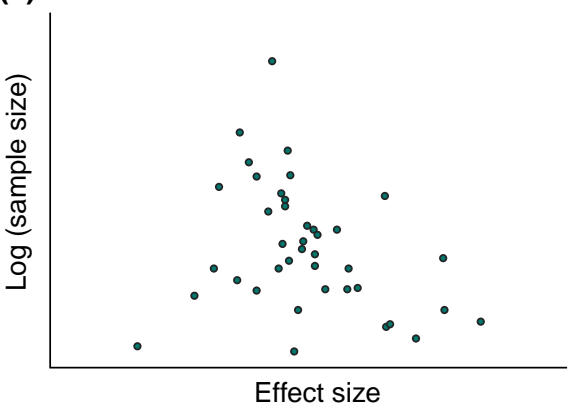

$\overline{\text { TRENDS in Ecology \& Evolution }}$

If effect sizes derive from a random sample of studies using similar research methods, a plot of sample size (or the standard error of the effect size) against effect size should reveal a funnel, with larger variance in values at small sample sizes and a decreasing variance with increasing sample sizea (Fig. la). If the true effect differs significantly from zero, but is small to intermediate, and publication is related to statistical significance (unless sample size is large), a decrease in the effect sizes of studies with increasing sample size should be expected (Fig. Ib). This occurs because the smaller the sample size the greater the estimated effect sizes must differ from zero to be statistically significant. So, if the true effect is moderately greater than zero, for smaller sample sizes only those estimates that, owing to sampling error, are larger than the true mean effect are likely to reach significance. Studies to the lower left or centre of Fig. Ib therefore go unpublished, except for the occasional study that is significantly smaller than zero owing to sampling error.

A funnel plot of the study of survivorship of males and expression of secondary sexual characters ${ }^{\mathrm{b}}$ showed a significant decrease in the variance of effect sizes as sample size increased. Using samples (or species) as the units of analysis did, however, suggest a slight skew towards more positive effect sizes when sample size was low (Fig. Ic).

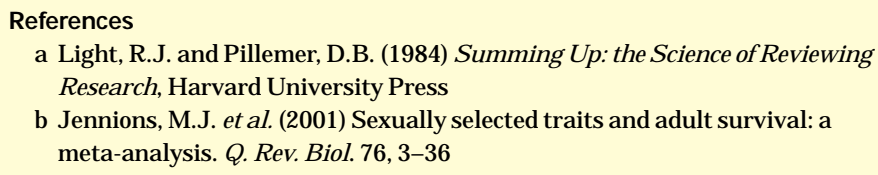

a second study, Nylenna et al. ${ }^{23}$ submitted two fictitious manuscripts for review to Scandinavians: one in English and the other in a Scandinavian language. TheE nglish manuscript was awarded consistently higher quality scores than was the nonEngl ish manuscript, although the contents were identical. Again, however, PB will only emergeif factors that influence a reviewer's decisions are correl ated with the strength of study findings.

Controlled investigation attempting to hideauthor identity from reviewers provided benefits in terms of paper quality in some ${ }^{24-26}$, but not all cases $^{27,28}$. F or example, articles from economics journals using double blind peer review werecited moreoften than werearticles from journals that only hide the reviewers' identity ${ }^{25}$. This failure might simply reflect thetendency for reviewers to guess author identity. Furthermore, unless both editors and reviewers are unaware of theauthors' names and institutions, the process is not truly anonymous. To avoid reviewer bias, some medical journals require reviewers to reveal their identity. This should reduce bias because reviewers are less likely to express unsubstantiated prejudices ${ }^{29}$ (but see Ref. 28). Obviously, revealing your identity as a reviewer could have other consequences, such as affecting the possibility of getting funding or acquiring a job, if a senior author can influencesuch decisions. Theusefulness of different peer-review approaches in ecol ogy and evolutionary biology deserves to beheld to referenda by members of academic societies.

\section{The written word: is there direct evidence for publication bias?}

Statistically significant results in theliteratureseem to be more common than would beexpected by chance ${ }^{16}$. Csada et al. ${ }^{15}$ reported that $91 \%$ of 1201 papers in 43 bi ol ogy journals had statistically significant supportivetests. Many similar results have been reported from themedical literature (Ref. 4). Direct comparison of published and unpublished studies is even more informative. For example, Dickersin et al. ${ }^{30}$ found that $55 \%$ of 767 publ ished medical studies showed a new treatment was better than a control, whereas only $14 \%$ of 178 unpublished studies found this effect. However, in two biological meta-analyses concerning sexual sel ection, neither Thornhill et al..$^{31}$ nor J ennions et al. ${ }^{32}$ found a significant difference in effect size adjusted for sample size between published and unpublished studies. In both thesestudies, extensive attempts to locate unpubl ished studies were made through contacts with scientists in the field in question. One caveat is that studies might be unpublished simply becausethey haveonly recently been completed. 


\section{Box 3. Rank comelation analysis of Begg and Mazumdar}

The rank correlation test of Begg and Mazumdara uses the Spearman rank correlation to investigate the relationship between standardized effect size and sample size or variance in effect size. The significance of the test can be found in standard statistical tables. In the studyb described in Box 2, there was no obvious heterogeneity in the funnel plot, using a graphical inspection. Applying the rank correlation test to the data revealed, however, that effect size decreased as sample size increased at both the study and species level of analysis (both: Spearman's $r=-0.317, P<0.05$ ). There were fewer than expected studies with negative effects at low sample sizes. Most biological meta-analyses appear to report the simple correlation between sample size and effect size without first standardizing effect size as recommended by Begg and Mazumdar. This could change conclusions. For the study ${ }^{b}$ described in Box 2 the nonstandardized correlations initially reported were Spearman's $r=-0.35$.

A linear regression approach to address the same question was reported by Allison et al.c ${ }^{c}$ This test is statistically more powerful, but requires several assumptions about the distribution of the data that might be unwarranted, for example, homoscedacity.

\footnotetext{
References

a Begg, C.B. and Mazumdar, M. (1994) Operating characteristics of a rank correlation test for publication bias. Biometrics 50, 1088-1101

b J ennions, M.J .et al. (2001) Sexually selected traits and adult survival: a meta-analysis. Q. Rev. Biol. 76, 3-36

C Allison, D.B. et al. (1996) Publication bias in obesity treatment trials. Int. J . Obes. 20, 445-447
}

\section{Dissemination bias: not all publications are equal}

Electronic searches are biased when the strength of the findings of located and unlocated publications differs. F or example, meta-analyses often rely on searching with language restrictions. Gregoireet al. ${ }^{35}$ showed than 28 out of 36 meta-analyses in medical journals had language restrictions, and at least two conclusions would have been altered by inclusion of nonE nglish literature. This is potentially a problem because nonE nglish-speaking authors might be more likely to publish nonsignificant results in their native language and significant findings in English ${ }^{3}$. More generally, nonsignificant results are published in journals with smaller circulation because of the previously described submission, review and editorial bias. These journals are less likely to be indexed in electronic data bases.

When compiling data for a meta-analysis, examination of referencelists of relevant papers is a standard practice. This could also produce a bias because biased use of available references is common. Thereis, for example, a bizarrefinding that the alphabetical position of surnames is correlated with citation rate ${ }^{36}$. M ore importantly, a study of a randomly selected citations from 283 research articles in psychology journals reveal ed that authors most commonly used citations to support their own argument, whereas study quality was only important for $2 \%$ of the citations ${ }^{37}$. A study of cholesterollowering medical trials revealed that supportive trials had a mean annual number of citations of 40 , whereas other trials only had 7.4 citations ${ }^{38}$, independent of journal of publication and samplesize of trials. Nationality of review authors might seriously bias citations, as shown in a study of reviews on chronic fatigue syndrome ${ }^{39}$. Among papers cited by authors from the USA, 72\% were from US journals, whereas $55 \%$ of citations by British authors were from British journals.

E ditors generally favour original and novel findings, and this decision might bias our view of how science normally functions. When last was a null result the cover story in Natureor in Science? A similar hunger for reporting positiveand novel studies is common in the news media, including general review journals, such as TREE. Newspaper coverage of a positive and a negative study on risk of cancer in relation to radiation published together in J AMA in 1991 showed that nine of 19 artides only covered the positiveresult. In the other ten articles, an average of 354 words was used for the positive study and 192 for the negative study ${ }^{40}$. Similarly, conferencetalks usually focus on significant findings: therefore, scientists are moreaware of these studies.

Ultimately, dissemination bias is only a dilemma when literature reviewers correlatethestrength of research findings with therate of encounter. The availableevidence suggests that studies with nonsignificant effects are encountered less often than are studies with significant effects. They aremore 


\section{Box 4. The 'trim and fill' method of testing and adjusting for publication bias}

The 'trim and fill' method of Duval and Tweediea is a nonparametric method testing and adjusting for publication bias (PB). The only assumption is a reliance on the symmetric distribution of effect sizes around the 'true' effect size in a funnel plot. The method assumes that it is the most extreme negative results that go unpublished. To find these studies, asymmetry in the funnel plot is detected. The extreme positive studies responsible for the asymmetry are then 'trimmed' off. An initial estimate of the mean effect size is then based on the symmetrical remainder. The original full data set is then re-examined to detect asymmetry around the new estimate of the mean effect size. Additional iterations are made until the result converges on the 'true' effect size. The missing, extreme negative results are then added by using the mirror image of the trimmed positive results, and this new distribution is used to derive final estimates of the mean effect size and its variance. Duval and Tweedie $e^{a}$ used three different estimators for the number of missing studies.

In the study of survivorship of males and expression of secondary sexual characters by J ennions et al. ${ }^{b}$ the number of missing studies can be obtained based on the method of Duval and Tweedie ${ }^{a}$. At the species level of analysis, the $R$ and $L$ estimators of the method indicated two and 11 missing studies for fixed-effects meta-analysis, but none for a random-effects metaanalysis. The mean effect size after trimming and filling for two or 11 missing studies respectively was $r=0.118(95 \% \mathrm{Cl} 0.090-0.146)$ and $r=0.072$ (95\% Cl $0.046-0.099$ ) (both $P<0.05$ ) compared with an original estimate of $0.125(95 \% \mathrm{Cl} 0.098-0.152)$. The conclusion is therefore robust even if it is assumed that 11 studies are missing owing to PB.

Several other methods (e.g. weighted distribution theory, general linear models and Bayesian modelling) have been derived to model PB (Ref. c). Unfortunately, these are not implemented in available software, and require considerable skills in statistical modelling. At present, 'trim and fill' appears to be the most parsimonious (and easiest) method with which to estimate the number of missing studies.

\footnotetext{
References

a Duvall, S. and Tweedie, R. (2000) Trim and fill: a simplefunnel-plot-based method of testing and adjusting for publication bias in meta-analysis. Biometrics 56, 455-463

b J ennions, M.J . et al. (2001) Sexually selected traits and adult survival: a meta-analysis. Q. Rev. Biol. 76, 3-36

c Song, F. et al. (2000) Publication and related biases. Health Technol. Assess. 4, 1-115
}

likely to be unpublished, published in low-circulation journals and published in languages other than English; and are less likely to becited and talked or written about.

\section{How to assess publication bias? The use of indirect methods}

PB can be assessed directly in a comparison of published and unpublished studies, or by following a cohort of studies from their inception. This is, however, a logistical challenge. Consequently, at least four types of indirect methods have been proposed to quantify PB. Unfortunately, the findings from these methods might have plausiblealternative explanations that are unrelated toPB. Conclusions should thus bemade cautiously ${ }^{31,32,41}$. There are currently no comparativeanalyses of the efficiency of different methods to identify PB and it is good practiceto useall of them. Consistency in findings provides greater confidence.

The fail-safe number of unpublished studies Among several availablemethods, calculating Rosenthal'S FAIL-SAFE NUMBER is the best known ${ }^{42}$. It estimates the number of unpublished studies, with a mean effect of zero, required to eliminatea significant overall effect size (Box 1). If the number of such studies is extremely large, it is unlikely that PB can alter the main conclusion of a meta-analysis regarding thesignificance of an effect ${ }^{43,44}$.

The use of thefail-safe method is problematic because of its emphasis on statistical rather than on biological significance. More importantly, if studies go unlocated because they reported results contrary to those in located studies, the 'true' fail-safe number is smaller than that based on an average effect of zero in unlocated studies.

\section{The funnel graph}

As a result of sampling error, small studies generatea greater range of findings than dolarger studies, resulting in a funnel-shaped relationship between effect sizeand sample size ${ }^{45}$. I f the probability of publication is greater for studies with statistically significant results, this skews or 'hollows out' the funnel shape of thegraph. If thetrue effect is small or moderate, but non-zero, and nonsignificant results tend not to be published, this creates a decrease in effect sizeas sample sizeincreases (Box 2). However, a skewed funNeL PLOT can becaused by factors other than PB because previous knowledge of effect sizes from pil ot studies, reduced samplesizes for certain species, choice of effect measures, chance and many other factors might cause asymmetric plots 31,46 .

\section{Effect size and sample size}

Several biological meta-analyses haveshown significant relationships between samplesize and effect size12,16,32. If thereis PB towards significant results, and the true effect size is small, but non-zero, effect size is expected to weaken as samplesize increases. Begg and Mazumdar ${ }^{47}$ proposed a distribution-free, rank correlation test to investigate this bias. Simulations show that the test is fairly powerful for largemeta-analyses with 75 studies, but only moderately so with 25 studies, requiring caution of interpretation (Box 3). 
Bias: systematic deviation of results from the truth, or processes leading to such deviation.

Dissemination bias: bias in the performance of research, publication, interpretation and review of scientific findings $\mathrm{s}^{\mathrm{a}}$. The accessibility of research findings to potential users depends on the direction or strength of these findings ${ }^{b}$

Effect size: a statistical measure of the magnitude of a relationship between two variables of interest. Typical measures of effect size in meta-analyses are the Pearson correlation coefficient, Hedges' $d$ (a measure of effect size in terms of normal standard deviates) or the odds ratio a,d.

Fail-safe number: The number of null results with a mean effect size of zero needed to change a given mean effect size adjusted for sample size into a nonsignificant result ${ }^{\mathrm{d}-\mathrm{g}}$.

Funnel plot: A graph illustrating the relationship between effect size and its variance. Traditionally, sample size or the standard error of the effect size is plotted against effect size.
Meta-analysis: A group of methodological techniques developed to synthesize a body of literature in a standardized way: (1) to obtain an estimate of the overall strength of a relationship; (2) to test for robustness of a common research finding : (3) to determine heterogeneity and its causes in a body of literature; and (4) to test for and investigate biases $\mathrm{c}, \mathrm{d}, \mathrm{h}$.

Publication bias: bias owing to the influence of research findings on submission, review and editorial decisions $\mathrm{a}, \mathrm{b}, \mathrm{i}$.

Submission bias: bias owing to the influence of research findings on the probability of submissionb.

\section{References \\ a Chalmers, I . (1990) Under-reporting research is scientific misconduct. J AMA 263, 1405-1408 \\ b Song, F. et al. (2000) Publication and related biases. Health Technol. Assess. 4, 1-115}

c Hedges, L.V. and Olkin, I . (1985) Statistical Methods for Meta-analysis, Academic Press

d Rosenthal, R. (1991) Meta-analytic Procedures for Social Research, Sage

e Rosenthal, R. (1979) The 'filedrawer problem' and tolerance for null results. Psychol. Bull. 86, 638-641

f Gleser, L.J . and Olkin, I. (1996) Models for estimating the number of unpublished studies. Stat. Med. 15, 2493-2507

g Gurevitch, J . and Hedges, L.V. (1999) Statistical issues in ecol ogical meta-analyses. Ecol ogy 80 , 1142-1149

h Arnqvist, G. and Wooster, D. (1995) Metaanalysis: synthesizing research findings in ecol ogy and evolution. Trends Ecol. Evol . 10, 236-240

i Dickersin, K. (1990) The existence of publication bias and risk factors for its occurrence. J AMA 263, 1385-1389
The trim and fill method

Thefeatures of thefunnel plot havebeen used to derive a nonparametric method of testing and adjusting for PB in meta-analysis ${ }^{48}$. This provides an estimate of the number of 'missing' studies owing to PB. Furthermore, the funnel plot is then used to obtain an estimate of effect size once these 'missing'studies are added (Box 4). This simpleiterative technique is based on estimating the number of studies in the asymmetrical, outlying part of the plot and then 'trimming'them off. The remaining studies arethen used to estimate moreaccurately the 'true' mean effect size. Thefull data set is then used to re-estimate the number of 'missing' studies based on asymmetry around the new estimate of mean effect size.

After just a few iterations, a robust estimate of the number of 'missing' studies and the mean effect size is obtained. Finally, thevariancein effect size is estimated from all availablestudies and the 'filled in' missing studies. The 'filled in' missing studies are simply the mirror-image counterparts of the trimmed-off studies with respect to the final estimated truemean effect.

\section{How can publication bias be avoided?}

What can bedoneto avoid the problem of PB? First, studies of PB might besubject to the same problems of publication bias as are other studies. Hence, indirect evidence of PB should be interpreted cautiously. As J ohnston and Breimer ${ }^{49}$ suggest: would studies of publication bias be published if no significant difference was found?

Meta-analysis might providea powerful tool for identifying PB. Although methods exist to correct estimates of effect size if $P B$ has been suggested, most assessments of potential bias areindirect. Alternative interpretations prevail so these methods are not particularly good 'remedies for publication bias'50. Instead, attempts to identify or adjust for PB should be used to investigate the robustness of conclusions, as is donein sensitivity analysis ${ }^{10,47}$.
Current knowledge of thecauses of PB suggests that investigators arethemain cause of bias becausethey fail to writeand submit (or resubmit) nonsignificant findings. Hopefully, with theincreasein onlinejournals, reduced competition for space and lower costs of publication will ensuretheeventual publication of all methodol ogically sound studies regardless of their actual research findings ${ }^{51,52}$.

\section{Conclusions}

Although problems of PB have been investigated in the medical and social sciences for morethan two decades, preliminary studies have only recently appeared in ecology and evolution ${ }^{11-16}$. Toassess theliterature, more information about potential biases is needed, in particular, more direct tests of PB comparing what has actually been published with all studies completed. Calls in society journals for members to register studies online would bea useful starting point. The fate of studies funded by major granting bodies could al so beexamined.

The problem of PB puts responsibility on the shoulders of editors, peer reviewers and authors to all ow fair publication of high-quality research, independent of research findings. Publication ethics deal with proper methods for analysing and presenting information in thescientific literature ${ }^{53}$ Based on theliteraturein medical sciences, it is potential authors in particular who should recognize their responsibility to publish their findings. Lack of publication by potential authors is even considered to bescientific misconduct by some scientists ${ }^{49,54}$.

Finally, when making reviews, authors should not only search electronic data bases, but al so check references and contact experts in the field. Although controversial in somescientificcircles, unpublished studies should beinduded to assess any PB. Although they can beunreliableguides to theexistence of genuine $\mathrm{PB}$, indirect methods do test the robustness of conclusions from meta-analyses and should al ways be used to test for PB. 


\section{References}

1 Coursol, A. and Wagner, E.E. (1986) Effect of positivefindings on submission and acceptance rates: a note on meta-analysis bias. Prof. Psychol. $17,136-137$

2 Dickersin, K. and Min, Y.I. (1993) Publication bias: the problem that won't go away. Ann. New York Acad. Sci. 703, 135-146

3 Sharp, D.W. (1990) What can and should be done to reduce publication bias? The perspective of an editor. J AMA 263, 1390-1391

4 Song, F. etal. (2000) Publication and related biases. Health Technol. Assess. 4, 1-115

5 Hedges, L.V. and Olkin, I . (1985) Statistical Methods for Meta-analysis, Academic Press

6 Rosenthal, R. (1991) Meta-analytic Procedures for Social Research, Sage

7 Arnqvist, G. and Wooster, D. (1995) Metaanalysis: synthesizing research findings in ecology and evolution. Trends Ecol. Evol. 10, 236-240

8 Glass, G.V. (1976) Primary, secondary, and metaanalysis of research. Educat. Res. 5, 3-8

9 Boisen, E. (1979) Testicular sizeand shape of $47, X Y Y$ and $47, X X Y$ men in a double-blind, double-matched population survey. Am. J . Hum. Genet. 31, 697-703

10 Song, F. and Gilbody, S. (1998) Increase in studies of publication bias coincided with increasing use of meta-analysis. Br. Med.J . 316, 471

11 Alatalo, R.V. et al. (1997) Heritabilities and paradigm shifts. Nature 385, 402-403

12 Gontard-Danek, M-C. and Møller, A.P. (1999) The strength of sexual selection: meta-analysis of bird studies. Behav. Ecol. 10, 476-486

13 Simmons, L.W. et al. (1999) Fluctuating paradigm. Proc. R. Soc. London B Biol. Sci. 266, 593-595

14 Poulin, R. (2000) Manipulation of host behaviour by parasites: a weakening paradigm? Proc. R. Soc. London B Biol. Sci. 267, 787-792

15 Csada, R.D. et al. (1996) The 'filedrawer problem ' of non-significant results: does it apply to biological research? Oikos 76, 591-593

16 Palmer, A.R. (2000) Quasireplication and the contract of error: lessons from sex ratios, heritabilities and fluctuating asymmetry. Annu. Rev. Ecol. Syst. 31, 441-480

17 Friedmann, S.H. and Karlsson, J .O.M. (1997)A novel paradigm. Nature 385,480

18 Kerr, S. et al. (1977) Manuscript characteristics which influenceacceptance for management and social science journals. Acad. Manage J . 20, 132-141
19 Mahoney, M.J . (1977) Publication prejudices: an experimental study of confirmatory bias in the peer review system. Cognit. Theor. Res. 1, 161-175

20 Ernst, R. and Resch, K.L. (1994) Reviewer bias a blinded experimental study. J . Lab. Clin. Med. 124, 178-182

21 Garfunkel, J .M. et al. (1994) Effect of institutional prestige on reviewers' recommendations and editorial decisions. J AMA 272, 137-138

22 Link, A.M. (1998) US and non-US submissions: an analysis of reviewer bias. J AMA 280, 246-247

23 Nylenna, M. etal. (1994) Multipleblinded reviews of the sametwo manuscripts: effects of referee characteristics and publication language. J AMA 272, 149-151

24 Fisher, M. et al. (1994) The effects of blinding on acceptance of research papers by peer review. J AMA 272, 143-146

25 Laband, D.N. and Piette, M.J . (1994) A citation analysis of theimpact of blinded peer review. I AMA 272, 147-149

26 McNutt, R.A. et al. (1990) The effect of blinding on the quality of peer-review. A randomised trial. J AMA 263, 1371-1376

27 Godlee, F. et al. (1998) Effect on the quality of peer review of blinding reviewers and asking them to sign their reports: a randomized controlled trial. J AMA 280, 237-240

28 Rooyen, S.V. et al. (1998) Effect of blinding and unmasking on the quality of peer review: a randomized trial. J AMA 280, 234-237

29 Walsh, E. et al. (2000) Open peer review: a randomised control trial. Br.J . Psychiatry 176, 47-51

30 Dickersin, K. et al. (1987) Publication bias and dinical trials. Cont. Clin. Trials 8, 343-353

31 Thornhill, R. et al. (1999) The biological significance of fluctuating asymmetry and sexual selection: A reply to Palmer. Am. Nat. 154, 234-241

32 J ennions, M.J . et al. (2001) Sexually selected traits and adult survival: a meta-analysis. Q. Rev. Biol. 76, 3-36

33 Bauchau, V. (1997) Is therea 'filedrawer problem' in biol ogical research? Oikos 79, 407-409

34 Stern, J.M. and Simes, R.J . (1997) Publication bias: evidence of delayed publication in a cohort study of clinical research projects. Br. Med.J . 315, 640-645

35 Gregoire, G. et al. (1995) Selecting the language of the publications included in a meta-analysis: is therea Tower of Babel bias? J . Clin. Epidemiol. 48, 159-163

36 Trengenza, T. (1997) Darwin a better namethan Wallace? Nature 385, 480
37 Shadish, W.R. et al. (1995) Author judgements about works they cite: threestudies from psychology journals. Soc. Stud. Sci. 25, 477-498

38 Ravnskov, U. (1992) Cholesterol lowering trials in coronary heart disease: frequency of citation and outcome. Br. Med. J . 305, 15-19

39 J oyce, J .et al. (1998) Reviewing the reviews. The example of chronic fatiguesyndrome. J AMA 280, 264-266

40 Koren, G. and Klein, N. (1991) Bias against negative studies in newspaper reports of medical research. J AMA 266, 1824-1826

41 Egger, M. et al. (1997) Language bias in randomised controlled trials published in English and German. Lancet 350, 326-329

42 Rosenthal, R. (1979) The 'filedrawer problem' and tolerancefor null results. Psychol. Bull. 86, 638-641

43 Gleser, L.J . and Olkin, I . (1996) Models for estimating the number of unpublished studies. Stat. Med. 15, 2493-2507

44 Gurevitch, J . and Hedges, L.V. (1999) Statistical issues in ecological meta-analyses. Ecol ogy 80 , 1142-1149

45 Light, R.J . and Pillemer, D.B. (1984) Summing Up: TheScience of Reviewing Research, Harvard University Press

46 Egger, M. et al. (1997) Bias in meta-analysis detected by a simple, graphical test. Br. Med.J . 315, 629-634

47 Begg, C.B. and Mazumdar, M. (1994) Operating characteristics of a rank correlation test for publication bias. Biometrics 50, 1088-1101

48 Duvall, S. and Tweedie, R. (2000) Trim and fill: A simplefunnel-plot-based method of testing and adjusting for publication bias in meta-analysis. Biometrics 56, 455-463

49 J ohnston, J .D. and Breimer, L.H. (1991) Publication bias. Br. Med. J . 337, 1102

50 Dickersin, K. (1990) Theexistence of publication bias and risk factors for its occurrence. J AMA 263, 1385-1389

51 Berlin, J.A. (1992) Will publication bias vanish in theage of onlinejournals? OnlineJ . Curr. Clin. Trials 1992 J ul. 8, Doc. No. 12 http://govt.ucsd.edu/newjour/o/msg01600.html

52 Song, F. et al. (1999) Therole of electronic journals in reducing publication bias. Med. Inform. Internet Med. 24, 223-229

53 Meslin, E.M. (1994) Toward an ethic in dissemination of new knowledgein primary care research. In Research Methods for Primary Care (Dunn, E.V., ed.), pp. 32-44, Sage

54 Chalmers, I. (1990) Under-reporting research is scientific misconduct.J AMA 263, 1405-1408

\section{Articles of interest}

Articles of ecological or evolutionary interest in recent issues of other Trends journals

Memory consolidation of Pavlovian fear conditioning: a cellular and molecular perspective, G.E. Schafe, K. Nader, H.T. Blair and J .E. LeDoux Trends in Neurosciences 24, 540-546 (September 2001)

Mechanisms of plant desiccation tolerance, F.A. Hoekstra, E.A. Golovina and J . Buitink Trends in Plant Science

6, 431-438 (September 2001)

Intracellular protozoan parasites and apoptosis: diverse strategies to modulate host-parasite interaction, C.G.K. Luder, U. Gross and M.F. Lopes Trends in Parasitology 17, 480-486 (October 2001)

Aspartyl proteinase genes from apicomplexan pararsites: evidence for the evolution of the gene structure, L. J ean, M. Long, J . Young, P. Péry and F. Tomley Trends in Parasitology 16, 491-498 (October 2001) 